\title{
Update on ropinirole in the treatment of Parkinson's disease
}

\author{
Holly A Shill \\ Mark Stacy \\ Sun Health Research Institute, \\ Sun City, AZ, USA; Duke University \\ and Medical Center, Durham, NC, USA
}

\begin{abstract}
Ropinirole is a dopamine agonist, approved for use to treat symptoms of early and advanced Parkinson's disease, is now available in a 24-hour formulation in addition to the immediate release version. This review discusses the mode of action of ropinirole and compares the pharmacokinetics of both formulations. Pivotal studies leading to the approval of both preparations are reviewed in terms of efficacy, dose range and side effects. Patient factors such as compliance are discussed in terms of the place for ropinirole in the armamentarium of Parkinson's disease therapies.
\end{abstract}

Keywords: ropinirole extended release, ropinirole immediate release Parkinson's disease

\section{Introduction}

For over a decade, ropinirole has been available for use in the management of early and advanced Parkinson's disease (PD). More recently, ropinirole extended release (Requip $^{\circledR} \mathrm{XL}^{\mathrm{TM}}$ ) has been approved for management of PD. We review the basic pharmacology of both forms of ropinirole and discuss the place of ropinirole extended release (24-hour) in the various stages of treatment of Parkinson's disease.

\section{Overview of pharmacology}

\section{Mode of action}

Ropinirole is a non-ergoline dopamine agonist with preferential affinity for the D2-like (D2, 3, 4) receptors. ${ }^{1}$ It has highest affinity at the D3 receptors which are concentrated in the limbic areas of the brain and may account for some of the neuropsychiatric effects. Lesser, but still significant, affinity is seen at the D2 receptor in the striatum accounting for the prominent benefits on the motor symptoms of PD. Negligible affinity is seen at $\alpha 2$ adrenoreceptors in the periphery and 5HT-1 receptor. Ropinirole has no affinity at the D1-like receptors, benzopdiazepine or GABA receptors.

The $\alpha 2$ adrenoceptor affinity may account for the cardiovascular effects seen with ropinirole as this effect can be blocked by the peripherally active agent domperidone., ${ }^{2,3}$ Tolerance to the cardiovascular effect is recognized, occurring after approximately one week of treatment. ${ }^{4}$

Ropinirole also has binding to lactotrophs in the anterior pituitary; ${ }^{5}$ however, the significance of this in the management of PD is likely minimal.

Putative neuroprotective effects in PD have been postulated given that ropinirole may have antioxidant effects in vivo, ${ }^{6}$ however, clinical studies report conflicting results, ${ }^{7,8}$ and the matter is still debated.

\section{Pharmacokinetics}

\section{Ropinirole immediate release}

After oral administration, ropinirole is rapidly absorbed with a typical $\mathrm{C}_{\max }$ of $1-2$ hours. $^{9,10}$ Oral bioavailability is approximately $50 \%$. Volume of distribution is 
large at approximately $7.5 \mathrm{~L} / \mathrm{kg}$. Plasma protein binding is approximately $40 \% .{ }^{11}$ Ropinirole is metabolized by the CYP-1A2 system. However, theophylline (CYP-1A2 inhibitor) administration showed no significant drug-drug interactions. ${ }^{12}$ Ciprofloxacin, another inhibitor of CYP-1A2, produced an increase in ropinirole $\mathrm{C}_{\max }$ by $60 \%$ without significant change in the clinical status of patients with PD. ${ }^{13}$ Hormone replacement therapy reduced clearance by $33 \%$ and advanced age had a small effect on reducing clearance. ${ }^{13}$ Taking ropinirole with food delays the time to $\mathrm{C}_{\max }$ by 2.6 hours but has no effect on the extent of absorption. ${ }^{14}$ Logically, dopamine antagonists such as typical dopamine receptor blocking drugs (eg, phenothiazines or metoclopramide) may reduce the efficacy of ropinirole.

Most of absorbed dose of ropinirole is cleared by the liver with only $10 \%$ being excreted unchanged in the urine. ${ }^{15}$ There are two metabolites, neither of which are pharmacologically active. Terminal half-life of ropinirole is about 6 hours (although there is a range of 2-10 hours) and is independent of dose. ${ }^{16}$

Ropinirole immediate release exhibits linear pharmacokinetics and given its profile, is typically administered as a three times a day medication. At steady state, the $\mathrm{C}_{\max }$ is roughly double what is seen in single dosing studies. The average peak to trough ratio is approximately $2: 1 .^{16}$

\section{Ropinirole 24-hour}

Ropinirole 24-hour is designed as a once daily long-acting formulation and comes in strengths that allow easy titration and switch from the immediate release formulation. In a comparison study ${ }^{17}$ with immediate release given every 8 hours, the 24 -hour ropinirole had a $\mathrm{T}_{\max }$ occurring 6 hours after administration, compared with 2 hours for the immediate release. After attainment of the $\mathrm{C}_{\max }$, the level slowly declined over the 24-hour period to a trough level roughly equivalent to the troughs seen with three times daily dosing of the immediate release. Dose normalized total concentration (AUCs) were approximately the same, whereas $\mathrm{C}_{\max }$ was about $12 \%$ lower with ropinirole 24 -hour. No significant food effects were seen. In clinical practice, if immediate release ropinirole is given with meals, the 12-hour gap between last evening dose and breakfast dosing, the differences between peak and trough levels may be as much as 5-fold, compared with the 2-fold change seen with ropinirole 24 -hour. Whether that is clinically relevant, remains uncertain.

\section{Efficacy}

\section{Ropinirole immediate release}

Ropinirole immediate release has a long established position in the management of Parkinson's disease. Short duration studies in early Parkinson's disease demonstrated a superior response to placebo for ropinirole monotherapy. ${ }^{18,19}$ Average total daily doses for these studies were $15.7 \mathrm{mg}$ /day and $6.54 \mathrm{mg}$ /day respectively. A 3-year study comparing ropinirole to bromocriptine (an ergoline dopamine agonist) showed a similar efficacy, safety and tolerability. ${ }^{20}$ A 5-year study of 268 subjects compared ropinirole to levodopa directly. ${ }^{7}$ The study monitored motor function as well as dopaminergic side effects. Ropinirole reduced the incidence of dyskinesia (45\% versus $20 \%$ ) as a primary motor complication of levodopa, as well as reduced the need for supplemental levodopa (total levodopa doses of $427 \mathrm{mg}$ versus $753 \mathrm{mg}$ ). Of the original 179 subjects who originally were assigned to receive ropinirole, $29(16 \%)$ remained in the study at 5 years on monotherapy. Although subjects experienced fewer dopaminergic motor complications in the ropinirole group, this was at the price of reduced efficacy in terms of both the motor examination and activities of daily living. The magnitude of the difference as measured by the UPDRS was 1.53 points for activities of daily living $(\mathrm{p}=0.08)$ and 4.48 for motor examination $(\mathrm{p}=0.008)$. It is important to recognize the limitation of this type of study, as only about $50 \%$ of subjects completed the study (regardless of arm) and the study constrained the use of supplemental medication to levodopa only which does not reflect current practice. This study also had a neuroimaging arm where a subset of subjects received ${ }^{18} \mathrm{~F}$-dopa positron emission tomography as an indirect marker of dopamine neuron loss. ${ }^{8}$ The 2-year study demonstrated that those originally assigned to ropinirole $(n=68)$ had a $14.1 \%$ decrease if fluorodopa uptake in the putamen compared with $22.9 \%$ decrease in those originally on levodopa $(n=59)$. Since this finding is in conflict with the findings of reduced clinical efficacy, there has been skepticism of whether this findings represents neuroprotection or may be explained by a pharmacological difference in the two drugs with respect to fluorodopa uptake. Most recently, 10-year follow-up data have been published demonstrating continued clinical benefit to ropinirole in a more naturalistic setting. ${ }^{21}$

Ropinirole has also been studied in more advanced PD. A placebo controlled study in patient not adequately controlled with levodopa showed a significant improvement in off time (35\% versus 13\%) and in daily levodopa reduction (242 mg versus $51 \mathrm{mg}$ ). ${ }^{22}$ A 16-week comparison study with bromocriptine showed that ropinirole added to levodopa therapy reduced the need for levodopa and had similar reduction in off time and improvement in the motor portion of the UPDRS compared to bromocriptine. ${ }^{23}$ 


\section{Ropinirole 24-hour}

Ropinirole 24-hour has been compared to placebo in advanced PD in a 24-week study in subjects with suboptimal control with levodopa. ${ }^{24}$ At week 24, mean daily dose was $18.8 \mathrm{mg} /$ day. Mean reduction in off time was 2.1 hours with ropinirole compared with 0.3 with placebo. Quality of life indicators, depression and sleep scales were also improved. A direct comparison to ropinirole immediate release has also been done with a similar design. The odds of having a $20 \%$ reduction in off time was significantly higher in 24-hour compared to immediate release ( $64 \%$ versus $51 \%$ ). Subjects were able to titrate to higher total daily doses, $18.6 \mathrm{mg}$ versus $10.4 \mathrm{mg}$, interpreted as better tolerability. Levodopa reductions were $162 \mathrm{mg} /$ day versus $113 \mathrm{mg} /$ day.

\section{Safety and tolerability Monotherapy with ropinirole immediate release}

The most common report side effects across studies were nausea, dizziness and somnolence, typically ranging from $10 \%$ to $20 \% .^{7,18-20}$ These side effects tend to peak during early titration and are diminished with longer term use, despite increase in dosing. Compared with levodopa, ropinirole produces more somnolence (27.4\% versus $19.1 \%)$, hallucinations (17.3\% versus $5.6 \%)$ and lower extremity edema (14.0\% versus 5.6\%). ${ }^{7}$ Nausea, dizziness and insomnia are similar between the two therapies. Sudden attacks of sleepiness resulting in motor vehicle accidents have been reported since approval, ${ }^{25}$ leading to a warning on the label. Additional label changes since approval include warnings concerning pathological gambling and other impulse control disorders (ICD). ${ }^{26}$ Recently, Weintraub et al summarized a prevalence study in which ICDs as a group were more common in patients treated with a dopamine agonist than non-dopamine agonist treated patients ( $17.1 \%$ versus $6.9 \%$ ). There was no difference in ICD frequency with pramipexole $(17.7 \%)$ versus ropinirole (15.5\%). ICDs seen were compulsive buying $(7.2 \%)$, gambling $(6.4 \%)$, binge eating (5.6\%) and compulsive sexual behavior $(4.4 \%){ }^{26}$

\section{Adjunctive therapy with ropinirole immediate release}

As adjunctive therapy to levodopa, ropinirole has a similar side effect profile as in monotherapy, with the exception of exacerbation of dyskinesia. Compared with placebo, dyskinesia are seen in $34 \%$ versus $13 \% .{ }^{22}$ Compared with bromocriptine, dyskinesia was seen $26 \%$ versus $14 \%$. However, in the long term efficacy trials in early PD, dyskinesia in subjects started on ropinirole was less than those started on levodopa, suggesting that the levodopa sparing factor may be important in reducing overall levels of dyskinesia?

\section{Ropinirole 24-hour}

Ropinirole 24-hour, not surprisingly, seems to have a similar adverse event profile as ropinirole immediate release. As adjunctive therapy, the most common side effects seen were dyskinesia (13\%), nausea (11\%), dizziness (8\%) and somnolence ( $7 \%) .{ }^{24}$ This compares with placebo where these same side effects were $4 \%, 3 \%$ and $4 \%$ respectively. Published data on comparison with immediate release ropinirole are somewhat lacking. A paper reviewing pharmacokinetics in small numbers of patients also discusses adverse effects and notes similar tolerability between ropinirole 24-hour and ropinirole immediate release with roughly the same total daily doses. A recent abstract suggests that ropinirole 24-hour can be titrated to higher daily doses in advanced $\mathrm{PD}^{27}$ suggesting better tolerability with a second abstract examining the same study supporting that conclusion..$^{28}$ No long term data are available to assess the frequency of impulse control disorder; however, one would expect little difference compared with immediate release.

Patients can be safely switched overnight from immediate release to ropinirole 24 -hour. ${ }^{17}$ With switching between dopamine agonists, rapid titration is preferable to slow titration in advanced Parkinson's disease. ${ }^{29}$

\section{Patient view}

In a Harris poll completed in 2003 (www.harrisinteractive. com), 228 patients with PD diagnosed more than 5 years were asked to rate their disease. Seventy-five percent reported at least 3 hours of off time per day. The average person took 19 pills per day, of which $60 \%$ were for PD. PD medication were taken an average of 4.5 times per day. Sixty-eight percent sometimes forgot to take medication; $41 \%$ did so regularly. Minimal side effects, improvement in daily on time and once-daily dosing were rated as first, third and seventh in terms of importance.

A survey of 250 PD patients on ropinirole immediate release showed that $67 \%$ of patients had missed doses (24\%) or were late on a dose (57\%) in the last week. ${ }^{30}$ Eighty-eight percent expressed interest in a once-daily formulation.

\section{Conclusion}

Ropinirole is regarded as a highly effective agent in treating the signs and symptoms of PD. While it is assumed the that 
the long acting compound (Requip ${ }^{\circledR} \mathrm{XL}^{\mathrm{TM}}$ ) will offer similar efficacy, the decreased pill burden and once daily therapy may improve patient compliance and as a result, provide additional symptomatic benefit. Furthermore, the reduced number of peak dose effects may have an impact on the drowsiness and orthostatic hypotension, thus improving tolerability to the agent.

\section{Disclosures}

The authors have no conflicts of interest to disclose.

\section{References}

1. Eden RJ, Costall B, Domeney AM, et al. Preclinical pharmacology of ropinirole (SK\&F 101468-A) a novel dopamine D2 agonist. Pharmacol Biochem Behav. 1991;38:147-154.

2. de Mey C, Enterling D, Meineke I, Yeulet S. Interactions between domperidone and ropinirole, a novel dopamine D2-receptor agonist. Br J Clin Pharmacol. 1991;32:483-488.

3. Eden RJ, Wallduck MS, Patel B, Owen DA. Autonomic and haemodynamic responses to SK\&F 101468 (ropinirole), a DA2 agonist, in anaesthetised cats. Eur J Pharmacol. 1990;175:333-340.

4. Parker SG, Raval P, Yeulet S, Eden RJ. Tolerance to peripheral, but not central, effects of ropinirole, a selective dopamine D2-like receptor agonist. Eur J Pharmacol. 1994;265:17-26.

5. Acton G, Broom C. A dose rising study of the safety and effects on serum prolactin of SK\&F 101468, a novel dopamine D2-receptor agonist. Br J Clin Pharmacol. 1989;28:435-441.

6. Iida M, Miyazaki I, Tanaka K, Kabuto H, Iwata-Ichikawa E, Ogawa N. Dopamine D2 receptor-mediated antioxidant and neuroprotective effects of ropinirole, a dopamine agonist. Brain Res. 1999;838:51-59.

7. Rascol O, Brooks DJ, Korczyn AD, De Deyn PP, Clarke CE, Lang AE. A five-year study of the incidence of dyskinesia in patients with early Parkinson's disease who were treated with ropinirole or levodopa. 056 Study Group. N Engl J Med. 2000;342:1484-1491.

8. Whone AL, Watts RL, Stoessl AJ, et al. Slower progression of Parkinson's disease with ropinirole versus levodopa: The REAL-PET study. Ann Neurol. 2003;54:93-101.

9. Kuzel MD. Ropinirole: a dopamine agonist for the treatment of Parkinson's disease. Am J Health Syst Pharm. 1999;56:217-224.

10. Boothman BR, Spokes EG. Pharmacokinetic data for ropinirole. Lancet. 1990;336:814.

11. Swagzdis JE, Wittendorf RW, DeMarinis RM, Mico BA. Pharmacokinetics of dopamine-2 agonists in rats and dogs. J Pharm Sci. 1986;75:925-928.

12. Thalamas C, Taylor A, Brefel-Courbon C, Eagle S, Fitzpatrick K, Rascol O. Lack of pharmacokinetic interaction between ropinirole and theophylline in patients with Parkinson's disease. Eur J Clin Pharmacol. 1999;55:299-303.

13. Kaye CM, Nicholls B. Clinical pharmacokinetics of ropinirole. Clin Pharmacokinet. 2000;39:243-254.
14. Brefel C, Thalamas C, Rayet S, et al. Effect of food on the pharmacokinetics of ropinirole in parkinsonian patients. Br J Clin Pharmacol. 1998;45:412-415.

15. Ramji JV, Keogh JP, Blake TJ, et al. Disposition of ropinirole in animals and man. Xenobiotica. 1999;29:311-325.

16. Hubble J, Koller WC, Atchison P, et al. Linear pharmacokinetic behavior of ropinirole during multiple dosing in patients with Parkinson's disease. J Clin Pharmacol. 2000;40:641-646.

17. Tompson DJ, Vearer D. Steady-state pharmacokinetic properties of a 24-hour prolonged-release formulation of ropinirole: results of two randomized studies in patients with Parkinson's disease. Clin Ther. 2007;29:2654-2666.

18. Adler CH, Sethi KD, Hauser RA, et al. Ropinirole for the treatment of early Parkinson's disease. The Ropinirole Study Group. Neurology. 1997;49:393-399.

19. Brooks DJ, Abbott RJ, Lees AJ, et al. A placebo-controlled evaluation of ropinirole, a novel D2 agonist, as sole dopaminergic therapy in Parkinson's disease. Clin Neuropharmacol. 1998;21:101-107.

20. Korczyn AD, Brooks DJ, Brunt ER, Poewe WH, Rascol O, Stocchi F. Ropinirole versus bromocriptine in the treatment of early Parkinson's disease: a 6-month interim report of a 3-year study. 053 Study Group. Mov Disord. 1998;13:46-51.

21. Hauser RA, Rascol O, Korczyn AD, et al. Ten-year follow-up of Parkinson's disease patients randomized to initial therapy with ropinirole or levodopa. Mov Disord. 2007;22:2409-2417.

22. Lieberman A, Olanow CW, Sethi K, et al. A multicenter trial of ropinirole as adjunct treatment for Parkinson's disease. Ropinirole Study Group. Neurology. 1998;51:1057-1062.

23. Im JH, Ha JH, Cho IS, Lee MC. Ropinirole as an adjunct to levodopa in the treatment of Parkinson's disease: a 16-week bromocriptine controlled study. J Neurol. 2003;250:90-96.

24. Pahwa R, Stacy MA, Factor SA, et al. Ropinirole 24-hour prolonged release: randomized, controlled study in advanced Parkinson disease. Neurology. 2007;68:1108-1115.

25. Frucht S, Rogers JD, Greene PE, Gordon MF, Fahn S. Falling asleep at the wheel: motor vehicle mishaps in persons taking pramipexole and ropinirole. Neurology. 1999;52:1908-1910.

26. Weintraub A, Koester J, Potenza MN, et al. Dopaminergic therapy and impulse control disorders in Parkinson's disease: Top line results of a cross sectional study of over 3,000 patients. In: Movement Disorder Society's 12th International Congress of Parkinson's disease and Movement Disorders. Chicago, Il; 2008.

27. Schapira AHV, Stocchi F, Hunter B, Giorgi L. Comparison of adjunctive ropinirole 24-hour prolonged release and immediate release in patietns with advanced Parkinson's disease: A per-protocol analysis ofthe PREPARED study. Mov Disord. 2008;23:S196-197.

28. Stocchi F, Tompson DJ, Giorgi L. Dosing of ropinirole 24-hour prolonged release in Parkinson's disease: Clinical trial data and relevance to clinical practice. Mov Disord. 2008;23:S214.

29. Goetz CG, Blasucci L, Stebbins GT. Switching dopamine agonists in advanced Parkinson's disease: is rapid titration preferable to slow? Neurology. 1999;52:1227-1229.

30. Edin HM, DeBenedetti DB, Hill CD. Adherence among Parkinson's disease patietns taking ropinirole immediate release at least thrice daily. Mov Disord. 2008;23:S344-345. 